\title{
A LINEAR, ALMOST PERIODIC EQUATION WITH AN ALMOST AUTOMORPHIC SOLUTION
}

\author{
RUSSELL A. JOHNSON
}

\begin{abstract}
We construct a scalar, first order, almost periodic ODE (*) $\dot{x}+A(t) x$ $=B(t)$ which admits bounded solutions, but no almost periodic solutions. Using this equation, we give an example of a two-dimensional, almost periodic system whose projective flow admits two minimal subsets, one of which is almost automorphic but not almost periodic. Finally, we show that some equation in the hull of (*) admits an almost automorphic, nonalmost periodic solution.
\end{abstract}

1. Introduction. We construct a scalar, first order, almost periodic ODE (*) $\dot{x}+A(t) x=B(t)$, which admits bounded solutions, but no almost periodic solutions. The function $A(t)$ was constructed by Conley and Miller in [1]. Using this equation, we give an example of a two-dimensional almost periodic system whose projective flow (see 2.3 below) admits two minimal subsets, one of which is almost automorphic [6] but not almost periodic. This answers in the affirmative a conjecture of the author in [4]. Then, we show that some equation in the hull of (*) admits an almost automorphic, nonalmost periodic solution. This answers questions $\mathbf{A}, \mathbf{H}$, and I posed by Fink [2, pp. 101, 168, 173]. Finally, we note that some equation in the hull of (*) has the following property: Every nontrivial solution to the corresponding homogeneous equation is bounded away from zero; however, the equation itself has a bounded solution, but no almost periodic solutions. This shows that Favard's theorem for linear inhomogeneous equations with almost perodic coefficients need not be true when the Favard property is not satisfied on the entire hull.

\section{Results.}

2.1. In $\$ \S 3$ and 4 , we will construct a linear, nonhomogeneous differential equation

$$
\dot{x}+A(t) x=B(t)
$$

with the following properties: (a) $A(t)=\lim _{n \rightarrow \infty} A_{n}(t)$ and $B(t)=\lim _{n \rightarrow \infty} B_{n}(t)$, where the limits are uniform on $\mathbf{R}$, and $A_{n}$ and $B_{n}$ have period $2^{n}(n>4)$. (b) $\int_{0}^{t} A(s) d s \rightarrow \infty$ as $t \rightarrow \infty$. (c) If $x(t)$ is the solution to (1) satisfying $x(0)=0$, then $x\left(2^{n}\right)=1 / 5(n>4, n$ odd); also, $|x(t)|<1$ for all $t \in \mathbf{R}$. Thus $x(t)$ is uniformly bounded. Moreover, $x\left(2^{n}\right)=0$ ( $n \geqslant 4, n$ even).

In the section, we prove that (1) has no almost periodic (a.p.) solutions (2.2), and state why it answers the questions discussed in the Introduction (2.3).

Received by the editors April 30, 1980.

AMS (MOS) subject classifications (1970). Primary 34A30, 34C99, 54H20.

(C) 1981 American Mathematical Society 0002-9939/81/0000-0258/\$02.75 


\subsection{TheOREM. Equation (1) admits no almost periodic solutions.}

Proof. Suppose (1) has an a.p. solution $\bar{x}(t)$. Then the frequency module of $\bar{x}(t)$ is contained in that of $A$. For, if not, then it is easily seen that (1) has an a.p. solution $\tilde{x}(t)$ such that $\tilde{x}(0) \neq \bar{x}(0)$. However, $(\tilde{x}(0)-\bar{x}(0)) \exp \left(-\int_{0}^{t} A(s) d s\right) \rightarrow 0$ as $t \rightarrow \infty(2.1(\mathrm{~b}))$, which is impossible unless the a.p. functions $\bar{x}$ and $\tilde{x}$ are identically equal. So the frequency module of $\bar{x}$ is contained in that of $A$. Hence $\lim _{n \rightarrow \infty} \bar{x}\left(2^{n}\right)$ exists (2.1(a)). However, if $x(t)$ is as in 2.1(c), then $\bar{x}(t)-x(t) \rightarrow 0$ as $t \rightarrow \infty$. By 2.1(c), we must have $\lim _{k \rightarrow \infty} \bar{x}\left(2^{2 k}\right)=0, \lim _{k \rightarrow \infty} \bar{x}\left(2^{2 k+1}\right)=1 / 5$. We have obtained a contradiction and proved Theorem 2.2.

2.3. Remarks. Consider the 2nd order ODE

$$
\dot{x}=\left[\begin{array}{cc}
(-1 / 2) A(t) & B(t) \\
0 & (1 / 2) A(t)
\end{array}\right] \equiv D(t) x \quad\left(x \in \mathbf{R}^{2}\right) .
$$

Let $\Omega$ be the hull of $D$ ( $=$ the uniform closure of the set of translates of $D$; see [15]). Write $\omega_{0} \equiv D \in \Omega$. It is well known that (2) induces, in a natural way, a flow on the projective bundle $\Sigma=\Omega \times \mathbf{P}^{1}$ [15]. Introduce polar coordinates $(r, \theta)$ in $\mathbf{R}^{2}$. Since $D$ is triangular, we may view $\Sigma$ as $\Omega \times\{\theta: 0<\theta<\pi\}$, where $\theta=0$ and $\theta=\pi$ are identified. Clearly $\Omega \times\{\theta=0\} \equiv M_{0}$ is a minimal subset of $\Sigma$.

Now express (2) in polar coordinates. It is easily seen that $\cot ^{-1} \theta(t)$ satisfies (1). Hence the orbit closure $\operatorname{cls}\left[\left(\omega_{0}, \pi / 2\right) \cdot t: t \in \mathbf{R}\right] \subset \Omega \times(0, \pi)$ contains a minimal set $M_{1}$ which, by 3.1, cannot be an a.p. minimal set. By 6.11 of [4], $M_{1}$ is an almost automorphic (a.a.) extension [6] of $\Omega$ which is not a.p. Thus the conjecture of [4] is true.

(b) From (a) and the definition of a.a. extension [ 6 , there is a point $\left(\omega_{1}, \theta_{1}\right) \in$ $M_{1}$ such that $M_{1} \cap\left(\left\{\omega_{1}\right\} \times[0, \pi]\right)=\left\{\left(\omega_{1}, \theta_{1}\right)\right\}$. (Such a point is an almost automorphic point of $M_{1}$.) Write $\left(\omega_{1}, \theta_{1}\right) \cdot t=\left(\omega_{1} \cdot t, \theta_{1}(t)\right)$. Then $\cot ^{-1} \theta_{1}(t)$ is an a.a. function of $t$ [6]. It is not a.p.; otherwise, $M_{1}$ would be an a.p. minimal set. It is a solution to

$$
\dot{x}+\tilde{A}(t) x=\tilde{B}(t),
$$

where $\tilde{A}$ resp. $\tilde{B}$ is a limit of translates of $A$ resp. $B$. So (3) is an a.p., scalar ODE with an almost automorphic, non-a.p. solution. This answers a question of Fink [2]. For further discussion, see [3].

(c) From [5], there is an equation of the form (3) (where again $\tilde{A}$ resp. $\tilde{B}$ is a limit of translates of $A$ resp. $B$ ) such that the homogeneous equation $\dot{x}+\tilde{A}(t) x=0$ satisfies $\inf _{t}|x(t)|>0$ unless $x(t) \equiv 0$. (Note this is equivalent to $\int_{0}^{t} \tilde{A}(s) d s<M<$ $\infty$ for all $t$.) Such an equation (3) admits bounded solutions, but no almost periodic solutions. It therefore has the Favard property, but does not satisfy the conclusion of Favard's Theorem.

3. The Conley-Miller function. We first review the example of Conley and Miller [1]. Choose numbers $a_{n}$ as follows: $a_{1}=1, a_{n}=a_{n-1}$ if $n$ is even; $a_{n}=(4 / 9)\left(a_{n-1}\right)$ if $n$ is odd (we choose the constant $B$ of [1] to be 4/9). Hence

$$
a_{n}=(2 / 3)^{n-2} \quad(n \text { even }) ; \quad a_{n}=(2 / 3)^{n-1} \quad(n \text { odd }) \text {. }
$$


Define a continuous, $2^{n}$-periodic functions $g_{n}(t)(n>3)$ as follows:

$$
\begin{aligned}
& g_{n}(t)= \begin{cases}0, & 0<t<2^{n-1}, \\
\text { linear, } & 2^{n-1}<t<2^{n-1}+\delta, \\
c_{n}, & 2^{n-1}+\delta<t<2^{n}-\delta, \\
\text { linear, } & 2^{n}-\delta<t<2^{n}, \\
0, & t=2^{n} \quad(n \text { odd })\end{cases} \\
& g_{n}(t) \equiv 0 \quad(n \text { even }) .
\end{aligned}
$$

Here

$$
\begin{gathered}
\delta=1 / 127 \\
c_{n}=(5 / 9) 2^{n}(2 / 3)^{n-3} /\left(2^{n-1}-\delta\right) \quad(n \text { odd }) .
\end{gathered}
$$

Define $A_{1}(t)=(2 / 5) \cdot g_{1}(t)$; then let

$$
A_{2}(t) \equiv A_{1}(t) ; \quad A_{n}(t)=A_{2}(t)-\sum_{i=3}^{n} g_{i}(t) \quad(n>3) ; \quad A(t)=\lim _{n \rightarrow \infty} A_{n}(t) .
$$

Thus $A_{n}(t) \equiv A_{n-1}(t)$ if $n$ is even, and $A(t)=A_{n+1}(t)=A_{n}(t)$ if $0<t<2^{n}$ for all $n$.

Conley and Miller show that

$$
\begin{aligned}
& \int_{0}^{t} A(s) d s \rightarrow \infty \quad \text { as } t \rightarrow \infty \\
& \int_{0}^{2^{n}} A(s) d s=2^{n} a_{n}=\begin{array}{ll}
4(4 / 3)^{n-2}, & n \text { even, } \\
2(4 / 3)^{n-1}, & n \text { odd; }
\end{array} \\
& \int_{2^{n}}^{2^{n+1}} A_{n+1}(s) d s=2^{n}\left(2 a_{n+1}-a_{n}\right)=(-1 / 4)(4 / 3)^{n} \quad(n \text { even }) .
\end{aligned}
$$

We need some additional facts about $A(t)$.

\subsection{Lemma. For $n>3, \int_{0}^{t} A_{n}(s) d s>a_{n} t$ for all $t>1$.}

Proof. Since $A_{n}$ is $2^{n}$-periodic, we can assume $0<t<2^{n}$.

The statement is true if $n=3$. Suppose it is true for some integer $n>3$. If $n$ is odd, then $A_{n+1}=A_{n}$ and $a_{n+1}=a_{n}$, so the statement holds with $n+1$ in place of $n$. Suppose $n$ is even. Then $h(t)=\int_{0}^{t} A_{n+1}(s) d s>a_{n+1} t$ for $t \in\left[0,2^{n}\right]$. Clearly $h(t)>a_{n+1} t$ for $t \in\left[2^{n}, 2^{n}+\delta\right]$. For $2^{n}+\delta<t<2^{n+1}-\delta$, one has

$$
\begin{aligned}
h(t) & >\left[a_{n+1}+\left(a_{n}-a_{n+1}\right)\right] t-c_{n+1}\left(t-2^{n}-\frac{1}{2} \delta\right) \\
& >a_{n+1} t \text { if } t<2^{n+1}\left(2^{n}+\frac{1}{2} \delta\right) /\left(2^{n}+\delta\right) .
\end{aligned}
$$

This last expression is $>2^{n+1}-\delta$, so $h(t)>a_{n+1} t$ on $\left[2^{n}, 2^{n+1}-\delta\right.$ ]. Finally, if $2^{n+1}-\delta<t<2^{n+1}$, then $h(t)$ is bounded below by a linear function of $t$ which is (i) $>a_{n+1} t$ if $t=2^{n+1}-\delta$; (ii) $=a_{n+1} t$ if $t=2^{n+1}$. This completes the proof.

3.2. LEMMA. Let $I_{1}=[0,2], I_{k}=\left[2^{k-1}, 2^{k}\right](k>1)$. If $n>4$, then

$$
\exp \left(-\int_{0}^{t} A_{n}(s) d s\right)<5 \cdot \sum_{i=k+1}^{n+1} \frac{1}{2^{i}} \quad \text { for } t \in I_{k}(1<k<n) \text {. }
$$


Proof. The statement is true if $n=4$. Suppose it is true for some $n>4$. If $t \in I_{k}$ $(1<k<n)$, then $\exp \left(-\int_{0}^{t} A_{n}(s) d s\right)<5 \sum_{i=k+1}^{n+1} 1 / 2^{i}<5 \sum_{i=k+1}^{n+2} 1 / 2^{i}$. Suppose $t$ $\in I_{n+1}$. Using 2.1 , we have $\int_{0}^{t} A_{n+1}(s) d s>a_{n+1} \cdot 2^{n}=(4 / 3)^{n}$. But $e^{-(4 / 3)^{n}}<$ $5 / 2^{n+2}$ for all $n>1$. The lemma is proved.

\subsection{Lemma. Define}

$$
\Delta_{n}=\exp \left(-\int_{7 \cdot 2^{n-3}}^{2^{n}} A_{n}(s) d s\right) \quad(n>3)
$$

Suppose $n \geq 4$ is even. Then $\left(1 / 5 \Delta_{n+1}\right) \exp \left(-\int_{7 \cdot 2^{n-2}}^{t} A_{n+1}(s) d s\right)<1 / 2^{n-1}+1 / 2^{n}$ for $t \in\left[7 \cdot 2^{n-2}, 31 \cdot 2^{n-4}\right]$.

Proof. Note that

$$
A_{n+1}(t)=\left\{\begin{array}{c}
A_{n-4}(t)-g_{n-1}(t)-g_{n+1}(t) \\
\quad \text { if } 7 \cdot 2^{n-2}<t<29 \cdot 2^{n-4} \text { or } 30 \cdot 2^{n-4}<t<31 \cdot 2^{n-4}, \\
A_{n-4}(t)-g_{n-3}(t)-g_{n-1}(t)-g_{n+1}(t) \\
\quad \text { if } 29 \cdot 2^{n-4}<t<30 \cdot 2^{n-4} \text { or } 31 \cdot 2^{n-4}<t<2^{n+1} .
\end{array}\right.
$$

Observe also that, by choice of $\delta$ and the assumption $n>4$,

$$
\frac{-195}{81}\left(\frac{4}{3}\right)^{n-4}>\int_{7 \cdot 2^{n-2}}^{2^{n+1}} A_{n+1}(s) d s>\frac{-197}{81}\left(\frac{4}{3}\right)^{n-4} \text {. }
$$

Let $h(t)=\int_{7 \cdot 2^{n-2}}^{t} A_{n+1}(s) d s$. If $7 \cdot 2^{n-2}<t<29 \cdot 2^{n-4}$, then

$$
h(t)>\left(a_{n-4}-c_{n-1}-c_{n+1}\right)\left(t-7 \cdot 2^{n-2}\right)>0 .
$$

If $29 \cdot 2^{n-4}<t<30 \cdot 2^{n-4}$, then

$$
h(t)>h\left(29 \cdot 2^{n-4}\right)+\left(a_{n-4}-c_{n-3}-c_{n-1}-c_{n+1}\right)\left(t-29 \cdot 2^{n-4}\right) \equiv V(t) .
$$

Hence $h(t)>V\left(30 \cdot 2^{n-4}\right)$, which is $>(-99 / 81)(4 / 3)^{n-4}$ by choice of $\delta$. If 30 . $2^{n-4}<t<31 \cdot 2^{n-4}$, then $h(t)>(-99 / 81)(4 / 3)^{n-4}$ also. Thus $\left(1 / 5 \Delta_{n+1}\right) e^{-h(t)}<$ $(1 / 5) \exp \left((-96 / 81)(4 / 3)^{n-4}\right)$ for $t \in\left[7 \cdot 2^{n-2}, 31 \cdot 2^{n-4}\right]$, and this is $<1 / 2^{n-1}+$ $1 / 2^{n}$ if $n>4$. The proof is completed.

3.4. Lemma. Suppose $n$ is even, $n>4$. If $31 \cdot 2^{n-4}<t<2^{n+1}$, then

$$
\frac{1}{5 \Delta_{n+1}} \exp \left(-\int_{7 \cdot 2^{n+1}}^{t} A_{n+1}(s) d s\right)<\frac{1}{5 \Delta_{n-1}} \exp \left(-\int_{31 \cdot 2^{n-4}}^{t} A_{n-1}(s) d s\right) .
$$

Proof. Let $\alpha(t)$ equal the left-hand side of the inequality, $\beta(t)$ the right-hand side. Then $\alpha(t)=C_{1} \exp \left(-\int_{31 \cdot 2^{n-4}}^{t} A_{n-1}(s) d s\right) \exp \left(\int_{31 \cdot 2^{n-4}}^{t} g_{n+1}(s) d s\right)$, where $C_{1}$ is a constant. Hence we have $\alpha(t) / \beta(t)=C_{2} \cdot \exp \left(\int_{31 \cdot 2^{n-4}}^{t} g_{n+1}(s) d s\right)$ for a constant $C_{2}$. Now, $\alpha\left(2^{n+1}\right)=\beta\left(2^{n+1}\right)=1 / 5$ and $g_{n+1}(s)>0$. Hence $\alpha(t)<\beta(t)$ if $31 \cdot 2^{n-4}<$ $t<2^{n+1}$, and the proof is finished.

4. The example. We turn now to our example. For $n>4$, we will consider equations

$$
\dot{x}+A_{n}(t) x=B_{n}(t),
$$

where $B_{n}$ is to be constructed. Let $x_{n}(t)$ be the solution to $(14)_{n}$ with $x_{n}(0)=0$. 
Let $n>4$ be even. Suppose that we have constructed a continuous function $B_{n}(t)$ such that $B_{n}$ and $x_{n}$ have the following properties:

$$
\begin{gathered}
B_{n} \text { has period } 2^{n} ; \\
x_{n}\left(2^{n}\right)=x_{n}\left(2^{n-2}\right)=\cdots=x_{n}\left(2^{4}\right)=0 ; \\
x_{n}\left(2^{n-1}\right)=\cdots=x_{n}\left(2^{5}\right)=1 / 5 ; \\
x_{n}\left(3 \cdot 2^{n-2}\right)=0 ; \\
x_{n}(t)>-\sum_{i=1}^{n} \frac{1}{2^{i}} \quad\left(0<t<2^{n}\right) ; \\
x_{n}(t)<\sum_{i=1}^{k} \frac{1}{2^{i}} \quad\left(0<t<2^{k}, 1<k<n\right) ; \\
x_{n}(t)+\frac{1}{5 \Delta_{n+1}} \exp \left(-\int_{7 \cdot 2^{n-2}}^{t+2^{n}} A_{n+1}(s) d s\right)<\sum_{i=1}^{n} \frac{1}{2^{i}} \quad \text { if } 3 \cdot 2^{n-2}<t<2^{n} .
\end{gathered}
$$

By (15) and (16), $x_{n}$ is $2^{n}$-periodic.

We will construct a continuous function $B_{n+2}(t)$ satisfying

$$
\begin{gathered}
B_{n+2}(t)=B_{n}(t) \quad\left(0<t<2^{n}\right) ; \\
\left|B_{n+2}(t)-B_{n}(t)\right|<3(2 / 3)^{n-3} \quad(t \in \mathbf{R}) ;
\end{gathered}
$$

also, (15)-(21) will hold with $n+2$ in place of $n$.

First, define a function $\tilde{B}^{n+1}(t)$ as follows: $\tilde{B}_{n+1}(t)=\dot{x}_{n}(t)+A_{n+1}(t) x_{n}(t)(t \in$ R). Then $\tilde{B}_{n+1}$ is $2^{n+1}$-periodic. Since $A_{n+2}=A_{n+1}$, we have $\tilde{B}_{n+1}(t)=\dot{x}_{n}(t)+$ $A_{n+2}(t) x_{n}(t)$. Also, $\left|\tilde{B}_{n+1}(t)-B_{n}(t)\right|<\left|A_{n+1}(t)-A_{n}(t)\right|<2(2 / 3)^{n-3}$ for all $t \in \mathbf{R}$ (use (19), (20), (6), and (7)).

Next, define a $2^{n+1}$-periodic function $B_{n+1}(t)$ by

$$
B_{n+1}(t)= \begin{cases}\tilde{B}_{n+1}(t), & 0 \leqslant t<7 \cdot 2^{n-2}-4, \\ \tilde{B}_{n+1}(t)+\gamma_{1}(t), & 7 \cdot 2^{n-2}-4<7 \cdot 2^{n-2}, \\ \tilde{B}^{n+1}(t), & 7 \cdot 2^{n-2}<t<2^{n+1}\end{cases}
$$

Here, $\gamma_{1}$ is a continuous, nonnegative function, supported on $\left[7 \cdot 2^{n-2}-4,7\right.$. $\left.2^{n-2}\right]$, such that

$$
\int_{0}^{7 \cdot 2^{n-2}} \gamma_{1}(s) \exp \left(-\int_{s}^{7 \cdot 2^{n-2}} A_{n+1}(r) d r\right) d s=\frac{1}{5 \Delta_{n+1}} .
$$

Since $A(t)<2$ for all $t$, we can and will take $\max \gamma_{1}(t)<1 / 5 \Delta_{n+1}<(2 / 3)^{n-3}$ (see (12) and (13), and recall $n>4$ ). Solving (14) $n+1$ explicitly, and using (18), we see that

$$
x_{n+1}(t)=\left\{\begin{array}{l}
x_{n}(t), \quad 0<t<7 \cdot 2^{n-2}-4 \\
x_{n}(t)+\eta_{1}(t), \quad 7 \cdot 2^{n-2}-4<t<7 \cdot 2^{n-2} \\
x_{n}(t)+\frac{1}{5 \Delta_{n+1}} \exp \left(-\int_{7 \cdot 2^{n-2}}^{t} A_{n+1}(s) d s\right), \quad 7 \cdot 2^{n-2}<t<2^{n+1}
\end{array}\right.
$$


where $0<\eta_{1}(t)<1 / 5 \Delta_{n+1}<1 / 2^{n+1}$ (see (12) and (13)). Hence

$$
\begin{aligned}
& x_{n+1}\left(2^{n+1}\right)=\frac{1}{5}, \quad x_{n+1}(t)=x_{n}(t) \quad\left(0<t<2^{n}\right) \\
& -\sum_{i=1}^{n+1} 1 / 2^{i}<x_{n+1}(t)<\sum_{i=1}^{n+1} 1 / 2^{i} \quad \text { (use (19)- (21)). }
\end{aligned}
$$

Here $0<t<2^{n+1}$. Now, define a $2^{n+2}$-periodic function $B_{n+2}$ by

$$
B_{n+2}(t)=\left\{\begin{array}{l}
B_{n+1}(t), \quad 0<t<3 \cdot 2^{n}-4, \\
\tilde{B}_{n+1}(t)-\gamma_{2}(t), \quad 3 \cdot 2^{n}-4<t<3 \cdot 2^{n}, \\
\tilde{B}_{n+1}(t), \quad 3 \cdot 2^{n}<t<2^{n+2} .
\end{array}\right.
$$

Here $\gamma_{2}$ is a nonnegative, continuous function supported on $\left[3 \cdot 2^{n}-4,3 \cdot 2^{n}\right]$; defining

$$
\eta_{2}(t)=\int_{0}^{t} \gamma_{2}(s) \exp \left(-\int_{s}^{t} A_{n+2}(r) d r\right) d s
$$

we require that

$$
\eta_{2}\left(3 \cdot 2^{n}\right)=\frac{1}{5} \exp \left(-\int_{2^{n+1}}^{3 \cdot 2^{n}} A_{n+2}(s) d s\right)
$$

Since $\int_{2^{n+1}}^{3 \cdot 2^{n}} A_{n+2}(s) d s=\int_{0}^{2^{n}} A_{n}(s) d s=4(4 / 3)^{n-2}$ (see (10)), we can and will assume that $\sup _{t} \gamma_{2}(t)<(2 / 3)^{n-3}$, and that $0<\eta_{2}(t)<1 / 2^{n+2}$ for $3 \cdot 2^{n}-4<t$ $<3 \cdot 2^{n}$. Solving $(14)_{n+2}$ explicitly, we obtain

$$
x_{n+2}(t)=\left\{\begin{array}{l}
x_{n+1}(t), \quad 0<t<2^{n+1}, \\
x_{n}(t)+\frac{1}{5} \exp \left(-\int_{2^{n+1}}^{t} A_{n+2}(s) d s\right), \quad 2^{n+1}<t<3 \cdot 2^{n}-4, \\
x_{n}(t)+\frac{1}{5} \exp \left(-\int_{2^{n+1}}^{t} A_{n+2}(s) d s\right)-\eta_{2}(t), \quad 3 \cdot 2^{n}-4<t<3 \cdot 2^{n}, \\
x_{n}(t), \quad 3 \cdot 2^{n}<t<2^{n+2},
\end{array}\right.
$$

We will show that conditions (15)-(23) hold with $n+2$ in place of $n$. By construction of $B_{n+2},(15),(22)$, and (23) are satisfied. By (22) and the formula for $x_{n+2}(t)$, (16), (17), and (18) hold. Since $x_{n+1}(t)>x_{n}(t)$ on $\left[0,2^{n+1}\right]$, and since $\eta_{2}(t)<1 / 2^{n+2},(19)$ holds.

Consider (20). By (24), $x_{n+2}(t)$ satisfies the required condition for $1<k<n+1$. By Lemma 3.2, the fact that $A_{n+2}=A_{n+1}$, and the assumption (20) on $x_{n}(t)$, we have $x_{n+2}(t)<\sum_{i=1}^{n+2} 1 / 2^{i}$ for $2^{n+1}<t<3 \cdot 2^{n}$. Since $x_{n+2}(t)<\sum_{i=1}^{n} 1 / 2^{i}<$ $\sum_{i=1}^{n+2} 1 / 2^{i}$ on $\left[3 \cdot 2^{n}, 2^{n+2}\right]$, the condition of (20) holds with $n+2$ in place of $n$.

Finally, consider (21). We must show that

$$
x_{n}(t)+\frac{1}{5 \Delta_{n+3}} \exp \left(-\int_{7 \cdot 2^{n}}^{t} A_{n+3}(s) d s\right)<\sum_{i=1}^{n+2} \frac{1}{2^{i}}
$$

for $7 \cdot 2^{n}<t<2^{n+3}$. If $7 \cdot 2^{n}<t<31 \cdot 2^{n-2}$, this follows from Lemma 3.3 and the assumption (20) on $x_{n}(t)$. Suppose that $31 \cdot 2^{n-2}<t<2^{n+3}$. Using Lemma 3.4 
and the assumption (21) on $x_{n}(t)$, we have

$$
\begin{aligned}
x_{n}(t)+\frac{1}{5 \Delta_{n+3}} \exp ( & \left.-\int_{7 \cdot 2^{n}}^{t} A_{n+3}(s) d s\right) \\
& <x_{n}(t)+\frac{1}{5 \Delta_{n+1}} \exp \left(-\int_{31 \cdot 2^{n-2}}^{t} A_{n+1}(s) d s\right) \\
& =x_{n}(t)+\frac{1}{5 \Delta_{n+1}} \exp \left(-\int_{7 \cdot 2^{n-2}}^{t-24 \cdot 2^{n-2}} A_{n+1}(s) d s\right) \\
& <\sum_{i=1}^{n} \frac{1}{2^{i}}<\sum_{i=1}^{n+2} \frac{1}{2^{i}} .
\end{aligned}
$$

Hence (21) holds for $x_{n+2}(t)$.

We can now construct the ODE promised in the Introduction. Let $x_{4}(t)$ be any continuously differentiable function of period $2^{4}=16$ satisfying (16)-(21) with $n=4$ (such a function exists; see Lemma 3.3). Define $B_{4}(t)=\dot{x}_{4}(t)-A_{4}(t) x_{4}(t)$. Then $B_{4}$ has period $2^{4}=16$, so (15) holds with $n=4$. By the preceding discussion, we may inductively construct functions $B_{n}(t)\left(n>4, n\right.$ even) such that $B_{n}$ and the solution $x_{n}$ to $(14)_{n}$ satisfying $x_{n}(0)=0$ satisfy (15)-(23) for all even $n>4$. Let $B(t)=\lim _{n \rightarrow \infty} B_{n}(t)$. By (15) and (23), $B$ is a.p. with frequency module contained in (actually, equal to) that of $A(t)$. Let $x(t)$ satisfy $x(0)=0$ and

$$
\dot{x}+A(t) x=B(t) \text {. }
$$

Then $x(t)=\lim _{n \rightarrow \infty} x_{n}(t)$. By (19) and (20), $|x(t)|<1$ for all $t \in \mathbf{R}$ (recall each $x_{n}$ is $2^{n}$-periodic); i.e., $x(t)$ is bounded. By (16), (17), and (22), 2.1(c) holds.

\section{REFERENCES}

1. C. C. Conley and R. A. Miller, Asymptotic stability without uniform stability: almost periodic coefficients, J. Differential Equations 1 (1965), 333-336.

2. A. M. Fink, Almost periodic differential equations, Lecture Notes in Math., vol. 377, Springer-Verlag, Berlin and New York, 1974.

3. R. Johnson, Bounded solutions of scalar, almost periodic differential equations, Illinois J. Math. (to appear).

4. Equations 37 (1980), 184-205.

5. R. J. Sacker and G. R. Sell, A spectral theory for linear differential systems, J. Differential Equations 27 (1978), 320-358.

6. W. A. Veech, Point-distal flows, Amer. J. Math. 92 (1970), 205-242.

Department of Mathematics, University of Southern Calufornia, los Angeles, California 90007 